This item was submitted to Loughborough's Research Repository by the author.

Items in Figshare are protected by copyright, with all rights reserved, unless otherwise indicated.

\title{
Exploiting multi-user diversity in wireless LANs with channel-aware CSMAVA
}

PLEASE CITE THE PUBLISHED VERSION

http://dx.doi.org/10.1109/PIMRC.2015.7343486

PUBLISHER

(C) IEEE

VERSION

AM (Accepted Manuscript)

LICENCE

CC BY-NC-ND 4.0

\section{REPOSITORY RECORD}

Wang, Xiaowei, Mahsa Derakhshani, and Tho Le-Ngoc. 2019. "Exploiting Multi-user Diversity in Wireless Lans with Channel-aware CSMA/CA”. figshare. https://hdl.handle.net/2134/21338. 


\title{
Exploiting Multi-User Diversity in Wireless LANs with Channel-Aware CSMA/CA
}

\author{
Xiaowei Wang*, Mahsa Derakhshani ${ }^{\dagger}$, Tho Le-Ngoc* \\ ${ }^{*}$ Department of Electrical \& Computer Engineering, McGill University, Montreal, QC, Canada \\ ${ }^{\dagger}$ Department of Electrical \& Computer Engineering, University of Toronto, Toronto, ON, Canada \\ Email: xiaowei.wang@mail.mcgill.ca; mahsa.derakhshani@utoronto.ca; tho.le-ngoc@mcgill.ca
}

\begin{abstract}
This paper presents a channel-aware access scheme for Carrier Sense Multiple Access with Collision Avoidance (CSMA/CA) aiming to take advantage of multi-user diversity and improve throughput, while supporting distributed and asynchronous operation. By dynamically adjusting the contention window of each station (STA) according to its channel state, this method prioritizes STAs that gain most from using a channel, and hence, enhances channel utilization in comparison with a simple random access scheme. To model the proposed Adaptive CSMA/CA (A-CSMA/CA) protocol, a three-dimensional Markov chain is developed. With the aid of such model, performance of the proposed A-CSMA/CA is analytically studied in terms of saturation throughput. Furthermore, illustrative results confirm that A-CSMA/CA significantly improves the throughput, specifically in a large network.
\end{abstract}

\section{INTRODUCTION}

The IEEE 802.11-based wireless local area networks (WLANs) are becoming more popular and widely deployed around the world. One of the reasons for such success is the robust medium access control (MAC) protocol, employing Carrier Sense Multiple Access with Collision Avoidance (CSMA/CA) [1]. However, in comparison with contention-free scheduling MAC protocols, CSMA/CA degrades the channel utilization because of collision avoidance overhead and inevitable collisions among STAs. Since the MAC protocol mainly determines the efficiency in spectrum sharing, efforts have been exerted on optimizing IEEE 802.11 MAC operation to achieve higher efficiency and throughput.

An important approach to improve spectrum efficiency is to optimize backoff algorithm using instantaneous channel state information (CSI) of STAs, which can take advantage of multiuser diversity (MUD). Among such works that explore MUD, there are both centralized and distributed approaches proposed in the literature. In the centralized schemes (e.g., [2], [3]), AP is responsible for scheduling, and thus CSIs need to be fed back to the AP (e.g., through multicast RTS/CTS [2]) by STAs. Such schemes will add overheads to the protocol and degrade the performance. To exploit MUD in a distributed manner, there are many works focused on p-persistent CSMA (e.g., [4]) and non-persistent CSMA (e.g., [5]) rather than CSMA/CA. These works contribute to the understanding of how to use the locally available CSI and MUD in CSMA/CA. But, since p-persistent and non-persistent CSMAs are different from CSMA/CA, it is difficult to implement the developed schemes while vendor products mostly use Distributed Coordination Function (DCF) protocol employing CSMA/CA.

Considering DCF protocol and CSMA/CA, there are few works aiming to achieve MUD in a distributed manner. For instance, to differentiate the STAs' channel access opportunities according to their CSIs, [6] proposes a scheme in which minimum contention window and frame size are adjusted according to their data rates. But, in this scheme, additional overheads are needed for the STAs to measure channel time of other STAs. Furthermore, [7] introduces an additional waiting duration, i.e., an inter-frame gap (IFG), that is adaptive to the channel state of STAs after the random backoff of DCF. However, the additional IFG requires much modification over the original DCF protocol. Moreover, [8] includes a binary refinement process into CSMA/CA before backoff mechanism to sift out only the STAs with good channel conditions. However, to implement such additional process, major modifications are required to harmonize it with DCF. In addition, the number of STAs must be known to achieve the best system performance.

Aiming to avoid major modifications over CSMA/CA-based $\mathrm{DCF}$, in this work, a channel-aware CSMA/CA is presented to benefit from MUD. In the proposed adaptive CSMA/CA (ACSMA/CA), prioritizing STAs with a good channel quality is based on adjusting the contention window independently by each STA, instead of centrally as in [2], [3]. Consequently, A-CSMA/CA can support fully distributed and asynchronous operation on STA-side. Since the contention window is adaptive to the backoff stage, the modification on the DCF protocol is minor as compared to the approaches in [6]-[8].

To evaluate the performance of A-CSMA/CA and study the impacts of specific system parameters on the saturation throughput, we develop a three-dimensional Markov chain modeling A-CSMA/CA protocol. With this model, the system throughput under different configurations are analyzed and compared with simulation results.

The remainder of this paper is organized as follows. Section II presents an overview of the system model. In Section III, the A-CSMA/CA scheme is introduced. Also, a three-dimensional Markov chain is developed to model the A-CSMA/CA protocol. Next, in Section IV, the saturation throughput of the proposed A-CSMA/CA is analyzed. Furthermore, Section V provides illustrative results to validate the throughput analysis and illustrate throughput improvement offered by ACSMA/CA comparing to the conventional CSMA/CA. Finally, 


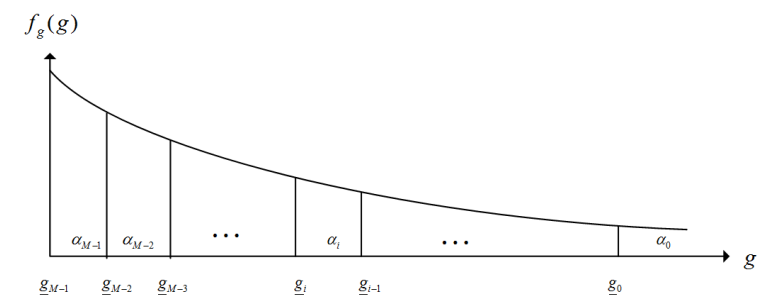

Fig. 1: Channel power gain distribution and range for AMC modes

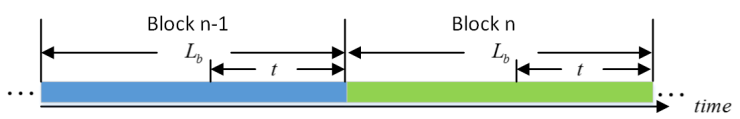

Fig. 2: Block fading channel model

Section VI presents the concluding remarks.

\section{SySTEM MODEL}

We consider an IEEE 802.11 WLAN consists of an Access Point (AP) and $N$ stations (STAs). We assume that the network is saturated, i.e., the STAs always have data for transmission.

\section{A. 802.11 Physical Layer}

In 802.11 WLANs, the modulation scheme and code-rate can be adjusted according to the channel state. An STA chooses its transmission mode based on its instantaneous signal-to-noise ratio (SNR) to guarantee a certain packet-errorrate (PER). For simplicity, in this paper, we assume that the CSI is available at the STAs.

Assume that $M$ physical modes can be used at the physical layer (including the mode with extremely low SNR where no transmission is possible). For simplicity of analysis, we assign an index $s(s=0,1, \ldots, M-1)$ to each mode, where a larger $s$ corresponds to a mode with lower SNR and data rate. To guarantee a minimum PER at each physical mode $s$, there is a lower bound $S N R_{s}$ on the received SNR at the receiver. Assuming that $g \in[0, \infty)$ is the channel power gain between an STA and the AP, the SNR of this link is $S N R=P g / \sigma^{2}$ where $P$ is the transmitted power and $\sigma^{2}$ is the noise power.

Given fixed $P$ and $\sigma^{2}, S N R_{s}$ corresponds to a lower bound of the channel power gain $\underline{g}_{s}$. For a distribution $f_{g}(g)$ of the channel power gain $g$, Figure 1 shows that the channel power gain region for each mode $s$ can be represented by $\left[\underline{g}_{s}, \underline{g}_{s-1}\right)$ $\left(g \underline{g}_{-1}=\infty\right)$. For any $s \in\{0,1, \ldots, M-1\}$, the probability distribution of each region can be calculated as follows

$$
\alpha_{s}=\mathbb{P}\left\{\underline{g}_{s} \leq g<\underline{g}_{s-1}\right\}=\int_{\underline{g}_{s}}^{\underline{g}_{s-1}} f_{g}(g) d_{g} .
$$

\section{B. Block Fading Channel Model}

We assume a block fading channel model, i.e., the channel power gain remains the same during a time block of length $L_{b}$ and varies independently from one block to another. Given a sequence of time $\left\{t_{1}, t_{2}, \ldots, t_{n}, \ldots\right\}$, the channel states $\left\{s_{1}, s_{2}, \ldots, s_{n}, \ldots\right\}$ form a discrete Markov chain.

If time $t_{n}$ and $t_{n+1}$ belong to different fading blocks of an STA, we say that it experienced a block transition. Given that $t_{n+1}-t_{n}=t$, the STA will experience a block transition with probability 1 if $t$ is lager than a block length. However, if $t$ is smaller than a block length, a block transition will happen only if $t_{n}$ falls in the last period of duration $t$ in a block, as shown in Figure 2. As a result, the probability of block transition from $t_{n}$ to $t_{n+1}$ can be computed as,

$$
w(t)= \begin{cases}\frac{t}{L_{b}}, & \text { if } t<L_{b} \\ 1, & \text { otherwise }\end{cases}
$$

Furthermore, for a time interval of $t$, the channel state transition probability $\theta_{s, s^{\prime}}^{t}$ (i.e., the probability that the channel state changes from $s$ to $s^{\prime}$ in a time duration of $t$ ) can be computed. Assuming $t_{n+1}-t_{n}=t$, then $\theta_{s, s^{\prime}}^{t}$ is the conditional probability that the channel state at time $t_{n+1}$ is $s_{n+1}=s^{\prime}$, given that the channel state at time $t_{n}$ is $s_{n}=s$. More specifically, if $s^{\prime}=s, \theta_{s, s^{\prime}}^{t}$ can be computed as the sum of the probability that no block transition happens from $t_{n}$ to $t_{n+1}(1-w(t))$ and the probability that a block transition happens with the channel state changing to $s^{\prime}\left(w(t) \alpha_{s^{\prime}}\right)$. If $s^{\prime} \neq s, \theta_{s, s^{\prime}}^{t}$ is simply $w(t) \alpha_{s^{\prime}}$. In summary,

$$
\theta_{s, s^{\prime}}^{t}= \begin{cases}1-w(t)+w(t) \alpha_{s^{\prime}}, & \text { if } s^{\prime}=s \\ w(t) \alpha_{s^{\prime}}, & \text { if } s^{\prime} \neq s\end{cases}
$$

Based on the definition of $w(t)$ in (1), $\theta_{s, s^{\prime}}^{t}$ becomes

$$
\theta_{s, s^{\prime}}^{t}= \begin{cases}1+\frac{t}{L_{b}}\left(\alpha_{s^{\prime}}-1\right) & \text { if } t<L_{b}, s^{\prime}=s \\ \frac{t}{L_{b}} \alpha_{s^{\prime}} & \text { if } t<L_{b}, s^{\prime} \neq s \\ \alpha_{s^{\prime}} & \text { if } t \geq L_{b}\end{cases}
$$

\section{Adaptive CSMA/CA And Markov Chain Model}

In this section, we introduce the adaptive CSMA/CA design and present a three-dimentional Markov chain to mathematically model the A-CSMA/CA protocol.

\section{A. Adaptive CSMA/CA Protocol}

To exploit the MUD, the basic idea of A-CSMA/CA is to give transmission priority to the STAs in good channel states by controlling its contention window. More specifically, the higher is the channel power gain of a STA, the smaller contention window is chosen by that STA. The smaller contention window leads to a smaller expected backoff time, with which the STA can get access to the channel with a higher probability.

The A-CSMA/CA protocol is built on DCF and preserves most of its characteristics, such as DIFS, SIFS, ACK, and the binary exponential backoff rules. A brief review of DCF can be found in [9]. The key differences of the proposed scheme compared with DCF are summarized as follows.

1) Adaptive Contention Window: In CSMA/CA, the contention window $C W$ of an STA is a function of the backoff stage $i$ (i.e., $C W=2^{i} C W_{\min }$ ). In A-CSMA/CA, we set the contention window adaptive to both the backoff stage and the instantaneous channel state $s$, i.e, $C W=f(i, s) C W_{\min }$. Whenever an STA finishes a transmission, it will adjust its backoff stage $i$ and adaptively set its contention window to be a function of $i$ and the channel state $s$. More specifically, if the data frame was successfully transmitted, the STA would 


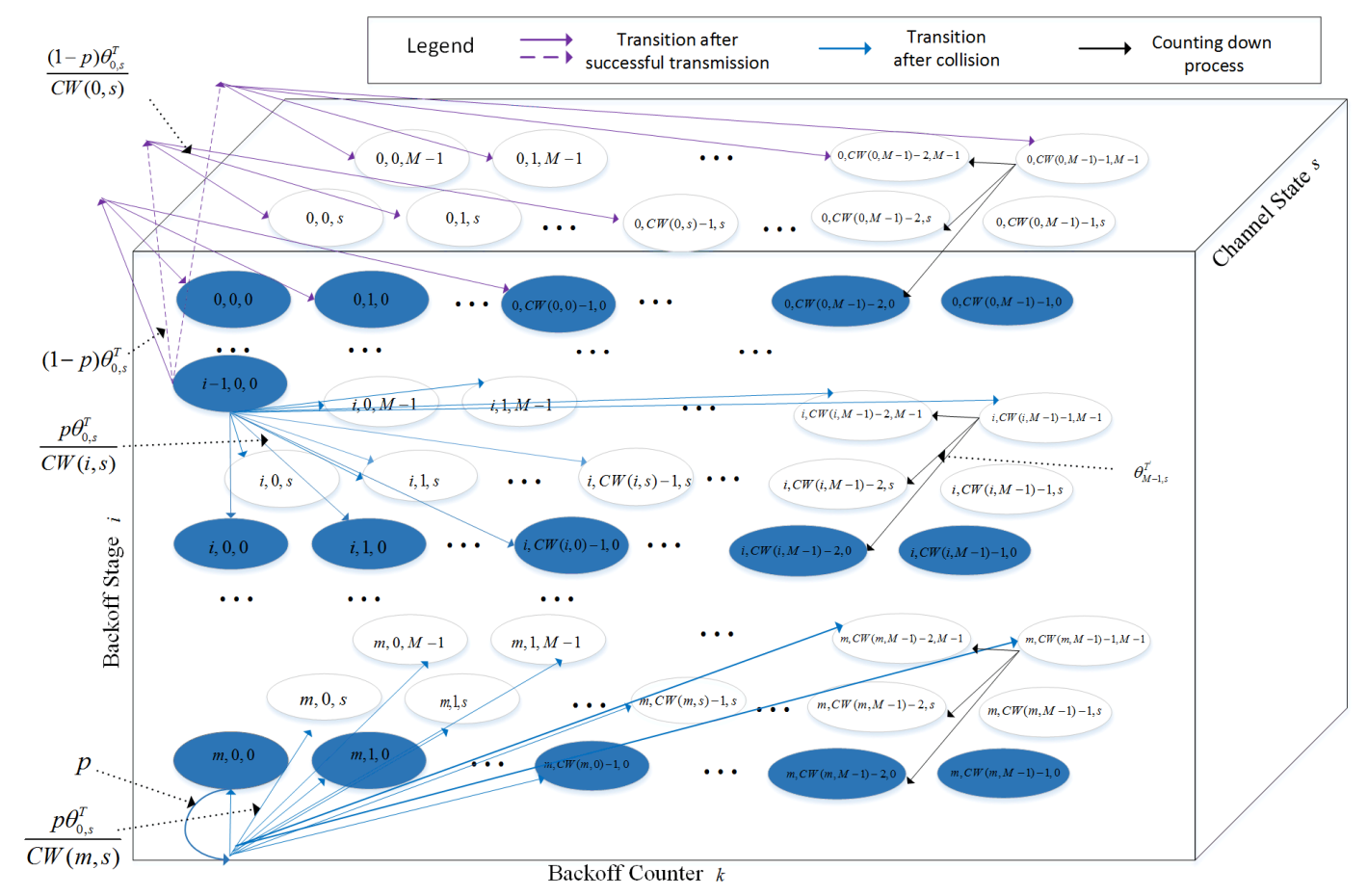

Fig. 3: Three-dimensional Markov chain model for one STA in A-CSMA/CA

set $i=0$ and $C W=f(i, s) C W_{\min }$. If a collision happened, the STA would set $i=\min (i+1, m)$ ( $m$ is the maximum number of backoff stages) and $C W=f(i, s) C W_{\min }$ where $f(i, s)$ is considered to be a general function of $i$ and $s$. For instance, $f(i, s)=2^{i} \beta^{s}$, where $\beta \in[1, \infty]$ is called channel backoff factor, implying that a lower channel power gain (i.e., having a larger $s$ ) results in a larger contention window and thus a lower priority for transmission.

2) Fixed Transmission Duration: As proposed in IEEE 802.11e amendment, we use the concept of transmission opportunity to reduce the backoff overhead. For each transmission opportunity, the STA transmits multiple back-to-back packets for a fixed duration of time $T$. The data volume (including header and payload) transmitted is $R_{s} \times T$ bits, where $R_{s}$ is the data rate for the current channel state $s$.

\section{B. Three-Dimensional Markov Chain Model for A-CSMA/CA}

To analyze the throughput of A-CSMA/CA protocol, we provide a three-dimensional Markov chain for the state transition of an STA based on the classic two-dimensional Markov chain model for DCF proposed by Bianchi in [10]. The additional dimension represents the channel state of an STA.

1) Markov Chain Model: Let define three stochastic processes for a given STA: $v(t)$ denotes its backoff stage, which takes a value from $\{0,1, \ldots, m\} ; b(t)$ denotes its backoff counter, which takes a value from $\{0,1, \ldots, C W(v(t), M-$ $1)-1\}$; and $c(t)$ denotes its channel state, which takes a value from $\{0,1, \ldots, M-1\}$. The three-dimensional Markov chain $\{v(t), b(t), c(t)\}$ can represent the state of a STA when it is in the A-CSMA/CA process. As in [10], we assume that the conditional collision probability for each transmission (seen by a STA) is constant, $p$, given that the number of STAs in the WLAN is fixed. According to the A-CSMA/CA protocol, the state transition of an STA can be summarized in Figure 3, where $\theta_{s, s^{\prime}}^{T}$ and $\theta_{s, s^{\prime}}^{T^{\prime}}$ are the channel transition probabilities for time durations of $T$ (the fixed transmission duration) and $T^{\prime}$ (the expected time for the STA to count down from $k+1$ to $k$, which will be calculated in the forthcoming section).

2) Transition Rules: We elaborate more on the three kinds of transitions that can happen in this Markov chain.

The first kind is transition after a successful transmission. As shown by the purple lines, when an STA starts a transmission at $\{i-1,0, s\}$, the total probability that this transmission succeeds is $1-p$. After the transmission duration of $T$, the channel state of this STA may change from $s$ to $s^{\prime}$ with probability $\theta_{s, s^{\prime}}^{T}$. If the STA is at the channel state $s^{\prime}$ once its transmission ends, according to the A-CSMA/CA protocol, it will uniformly pick a random backoff counter $k$ from $\left[0, C W\left(0, s^{\prime}\right)\right)$ for its next transmission. Thus, the STA moves to the state $\left\{0, k, s^{\prime}\right\}$ with probability $\frac{(1-p) \theta_{s, s^{\prime}}^{T}}{C W\left(0, s^{\prime}\right)}$.

The second kind is transition after a collision. As shown by the blue lines, when the STA starts a transmission from state $\{i-1,0, s\}$, the probability that this transmission fails due to collision is $p$. After the collision, the STA will backoff to stage $\min (i, m)$, while the channel state may change from $s$ to $s^{\prime}$ with probability $\theta_{s, s^{\prime}}^{T}$. If the STA is at the channel state $s^{\prime}$ once the collision ends, according to the A-CSMA/CA protocol, the STA will move to the state $\left\{\min (i, m), k, s^{\prime}\right\}$ with probability $\left.\frac{p \theta_{s, s^{\prime}}^{T}}{C W\left(\min (i, m), s^{\prime}\right)}, \forall k \in\left[0, C W\left(\min (i, m), s^{\prime}\right)\right)\right]$.

The third kind is transitions during the count down process. As shown by the black lines, when the STA is in a state $\{i, k, s\}$ where $k>0$, it decrements its backoff counter after the channel is sensed idle for a time-slot. Assuming that the 
$\left\{\begin{array}{l}b_{i, k, s}= \\ \sum_{s^{\prime}=0}^{M-1} \sum_{i^{\prime}=0}^{m} b_{i^{\prime}, 0, s^{\prime}}(1-p) \theta_{s^{\prime}, s}^{T} / C W(i, s) \\ 0 \\ \left.\sum_{i^{\prime}=0}^{M-1} b_{i^{\prime}, 0, s^{\prime}}(1-p) \theta_{s^{\prime}, s}^{T} / C W(i, s)+b_{i, k+1, s^{\prime}} \theta_{s^{\prime}, s}^{T^{\prime}}\right) \\ M-1 \\ \sum_{s^{\prime}=0}^{M-1}\left(\sum_{i^{\prime}=0}^{m} b_{i^{\prime}, 0, s^{\prime}}(1-p) \theta_{s^{\prime}, s}^{T} / C W(i, s)+b_{i, k+1, s^{\prime}} \theta_{s^{\prime}, s}^{T^{\prime}}\right) \\ \sum_{s^{\prime}=0}^{M-1} b_{i-1,0, s^{\prime}} p \theta_{s^{\prime}, s}^{T} / C W(i, s) \\ \sum_{s^{\prime}=0}^{M-1}\left(b_{i-1,0, s^{\prime}} p \theta_{s^{\prime}, s}^{T} / C W(i, s)+b_{i, k+1, s^{\prime}} \theta_{s^{\prime}, s}^{T^{\prime}}\right) \\ 0 \\ \sum_{s^{\prime}=0}^{M-1} b_{i, k+1, s^{\prime}} \theta_{s^{\prime}, s}^{T^{\prime}}, \\ \sum_{s^{\prime}=0}^{M-1}\left(b_{i-1,0, s^{\prime}} p \theta_{s^{\prime}, s}^{T} / C W(i, s)+b_{i, k+1, s^{\prime}} \theta_{s^{\prime}, s}^{T^{\prime}}\right) \\ \sum_{s^{\prime}=0}^{M-1}\left(b_{i-1,0, s^{\prime}}+b_{i, 0, s^{\prime}}\right) p \theta_{s^{\prime}, s}^{T} / C W(i, s) \\ \sum_{s^{\prime}=0}^{M-1}\left(\left(b_{i-1,0, s^{\prime}}+b_{i, 0, s^{\prime}}\right) p \theta_{s^{\prime}, s}^{T} / C W(i, s)+b_{i, k+1, s^{\prime}} \theta_{s^{\prime}, s}^{T^{\prime}}\right) \\ 0 \\ \sum_{s^{\prime}=0}^{M-1}\left(b_{i, k+1, s^{\prime}} \theta_{s^{\prime}, s}^{T^{\prime}}\right) \\ \sum_{s^{\prime}=0}^{M-1}\left(\left(b_{i-1,0, s^{\prime}}+b_{i, 0, s^{\prime}}\right) p \theta_{s^{\prime}, s}^{T} / C W(i, s)+b_{i, k+1, s^{\prime}} \theta_{s^{\prime}, s}^{T^{\prime}}\right)\end{array}\right.$

expected time needed to count down from $k$ to $k-1$ is $T^{\prime}$, the STA move to the state $\left\{i, k-1, s^{\prime}\right\}$ with probability $\theta_{s, s^{\prime}}^{T^{\prime}}$.

\section{Throughrut Analysis}

Based on the proposed three-dimentional Markov chain in Section III-B, here we analyze the throughput of an STA that follows the A-CSMA/CA protocol. The throughput is defined as the ratio of the expected number of bits transmitted in a general time slot to the expected duration of a general time slot. The fixed probability that an STA spends in each state at the stationary point of the Markov chain is computed in Section IV-A. Based on these probabilities, the probability that an STA will start a transmission in each channel state can be computed. With these transmission probabilities, the expected amount of information transmitted in a general time slot and the duration of a general time slot is calculated in Section IV-B to get the throughput of an STA.

A. Transmission Probability

1) Estimation of $T^{\prime}$ : As discussed earlier, $T^{\prime}$ denotes the expected time required for an STA to count down from $k$ to $k-1$. Since an STA has to freeze its backoff counter once the channel is sensed busy while counting down, $T^{\prime}$ is not simply equal to an idle time-slot.

Following the A-CSMA/CA protocol, the channel is sensed busy if there is a successful transmission or a collision. In either case, the channel will be busy for a time duration of $T$. Since the STA will only count down when the channel is idle and freeze its backoff counter when the channel is busy, $T^{\prime}$ can be computed as,

$$
\frac{T^{\prime}}{T_{\mathrm{b}}}=\frac{P_{\text {idle }} T_{\mathrm{b}}+\left(1-P_{\text {idle }}\right) T}{P_{\text {idle }} T_{\mathrm{b}}}
$$

$$
\begin{aligned}
& \forall i=0, s=M-1, k=C W(i, s)-1 \\
& \forall i=0, s=M-1, k=0: C W(i, s)-2 \\
& \forall i=0, s=0: M-2, k=C W(i, M-1)-1 \\
& \forall i=0, s=0: M-2, k=C W(i, s): C W(i, M-1)-2 \\
& \forall i=0, s=0: M-2, k=0: C W(i, s)-1 \\
& \forall i=1: m-1, s=M-1, k=C W(i, s)-1 \\
& \forall i=1: m-1, s=M-1, k=0: C W(i, s)-2 \\
& \forall i=1: m-1, s=0: M-2, k=C W(i, M-1)-1 \\
& \forall i=1: m-1, s=0: M-2, k=C W(i, s): C W(i, M-1)-2 \\
& \forall i=1: m-1, s=0: M-2, k=0: C W(i, s)-1 \\
& \forall i=m, s=M-1, k=C W(i, s)-1 \\
& \forall i=m, s=M-1, k=0: C W(i, s)-2 \\
& \forall i=m, s=0: M-2, k=0: C W(i, M-1)-1 \\
& \forall i=m, s=0: M-2, k=C W(i, s): C W(i, M-1)-2 \\
& \forall i=m, s=0: M-2, k=0: C W(i, s)-1
\end{aligned}
$$

where $T_{\mathrm{b}}$ is the duration of an idle backoff time-slot and $P_{\text {idle }}$ is the probability that channel sensed idle in a general timeslot by a given STA. Considering that each STA transmits in a general time-slot with probability $\tau, P_{\text {idle }}=(1-\tau)^{N-1}$. Based on (5), $T^{\prime}$ can be calculated as

$$
T^{\prime}=T_{\mathrm{b}}+\left((1-\tau)^{1-N}-1\right) T
$$

2) Stationary Distribution: Let $b_{i, k, s}=\lim _{t \rightarrow \infty} P\{v(t)=$ $i, b(t)=k, c(t)=s\}, \forall\{i, k, s\}$ be the stationary distribution of the presented Markov chain. Since the STA will start a transmission in the states $\{i, 0, s\}$. The transmission probability of each STA at the channel state $s$ is

$$
\tau_{s}=\sum_{i=0}^{m} b_{i, 0, s}, \quad \forall s \in\{0,1, \ldots, M-1\} .
$$

Subsequently, the total transmission probability $\tau$ is

$$
\tau=\sum_{s=0}^{M-1} \tau_{s}=\sum_{s=0}^{M-1} \sum_{i=0}^{m} b_{i, 0, s}
$$

Thus, aiming to find $\tau$, we need to calculate the stationary distribution of the Markov chain, i.e., $b_{i, k, s}$. Since at the stationary point, the incoming probability flow and outgoing probability flow for each state has to be equal to make the distribution probability constant. Each $b_{i, k, s}$ can be represented by a linear equation of all other $b_{i^{\prime}, k^{\prime}, s^{\prime}}$ as in (4).

Furthermore, by the normalization condition, we have,

$$
\sum_{s=0}^{M-1} \sum_{i=0}^{m} \sum_{k=0}^{C W(i, M-1)-1} b_{i, k, s}=1 .
$$

Given the fixed conditional collision probability $p$ and transmission probability $\tau$, (4) and (9) create a system of $\sum_{s=0}^{M-1} \sum_{i=0}^{m} C W(i, M-1)+1$ linear equations in $\sum_{s=0}^{M-1} \sum_{i=0}^{m} C W(i, M-1)$ variables (i.e., $\left.b_{i, k, s}\right)$. By solv- 
ing this system of linear equations, we can derive $b_{i, k, s}(\tau, p)$ as functions of $\tau$ and $p$.

The conditional collision probability $p$ can also be expressed as a function of $\tau$. By the definition, $p$ is the collision probability that any transmission experiences. Thus, it is the probability that at least one of the remaining $(N-1)$ STAs (except the transmitting STA) starts a transmission. Mathematically,

$$
p=1-(1-\tau)^{N-1} .
$$

Consequently, (8) and (10) represent a system of two nonlinear equations in $p$ and $\tau$ for a fixed number of STAs in the WLAN. By solving these equations numerically, $\tau$ and $p$ can be derived. Then, by substituting $\tau$ and $p$ back into $b_{i, k, s}(\tau, p)$, the stationary transmission probability in each channel state, i.e., $\tau_{s}$, can also be computed.

\section{B. Saturation Throughput}

In [10], the saturation throughput for CSMA/CA-based DCF is defined and calculated as the number of successfully delivered information bits per second. Mathematically, the throughput of an STA can be expressed as

$$
T=\frac{P_{\mathrm{s}} \mathbb{E}[I]}{\left(1-P_{\mathrm{tr}}\right) T_{\mathrm{b}}+P_{\mathrm{s}} T_{\mathrm{s}}+\left(P_{\mathrm{tr}}-P_{\mathrm{s}}\right) T_{\mathrm{c}}}
$$

where $P_{\mathrm{s}}$ is the probability that a successful transmission occurs in a general time-slot, $P_{\text {tr }}$ is the probability that at least one transmission, either successful or not, happens in a general time-slot, $T_{\mathrm{s}}$ and $T_{\mathrm{c}}$ are the average time that the channel is sensed busy because of a successful transmission and a collision, respectively. Furthermore, $\mathbb{E}[I]$ is the expected amount of payload information transmitted in a successful transmission. More specifically,

$$
\mathbb{E}[I]=T \mathbb{E}[R]-H=T \frac{\sum_{s=0}^{M-1} R_{s} \tau_{s}}{\sum_{s=0}^{M-1} \tau_{s}}-H
$$

where $\mathbb{E}[R]$ denotes the expected data rate, $R_{s}$ is the data rate can be offered at the channel state $s, \tau_{s}$ is the transmission probability at the channel state $s$ calculated in (7), and $H$ is the size of PHY and MAC header.

In the saturation throughput expression (11), the numerator represents the average amount of information bits that successfully transmitted in a general time-slot, i.e., $P_{\mathrm{s}} \mathbb{E}[I]$. In the denominator, the average length of a general time-slot is presented. In particular, a general time-slot is either idle with probability $\left(1-P_{\text {tr }}\right)$, it contains a successful transmission with probability $P_{\mathrm{s}}$, or it contains a collision with probability $P_{\mathrm{c}}=P_{\mathrm{tr}}-P_{\mathrm{s}}[10]$. To analyze the saturation throughput in (11), we first need to define $P_{\mathrm{s}}$ and $P_{\mathrm{tr}}$.

In a general slot, the probability that there is at least one transmission in the network is

$$
P_{\mathrm{tr}}=1-(1-\tau)^{N} .
$$

A transmitted data frame will be received successfully, if exactly one STA transmits on the channel. Thus, the probability of successful transmission (i.e., $P_{\mathrm{s}}$ ) becomes

$$
P_{\mathrm{s}}=N \tau(1-\tau)^{N-1}
$$

Based on (11), to specifically compute the saturation throughput, it is required to specify the values of $T_{\mathrm{s}}$ and $T_{\mathrm{c}}$. As in [10], Assuming that $\delta$ denotes the propagation delay, we have

$$
\begin{aligned}
& T_{\mathrm{s}}=T+\delta+S I F S+A C K+\delta+D I F S \\
& T_{\mathrm{c}}=T+\delta+D I F S
\end{aligned}
$$

\section{ILlustrative Results}

To evaluate the accuracy of the Markov chain model and the performance of the proposed protocol, we implement the A-CSMA/CA and CSMA/CA protocols in Matlab.

The MAC layer parameters are set as follows: $T_{b}=9 \mu \mathrm{s}$, $\delta=1 \mu \mathrm{s}, T=8 \mathrm{~ms}$, SIFS $=10 \mu \mathrm{s}$, DIFS $=30 \mu \mathrm{s}$, ACK $=40 \mu s, H=400$ bits. The channel bandwidth $B=20 \mathrm{MHz}$. We set SNR to $10 \mathrm{~dB}$ for all STAs. Furthermore, the channel power gains $g_{j}$ are randomly generated according to the Rayleigh distribution assuming $\mathbb{E}_{\mathrm{g}}\left[g_{j}\right]=1$.

The 802.11a physical layer is used to determine the transmission rate for each STA. Table I details the data rates and corresponding SNR ranges. Each data rate corresponds to a physical mode in the Markov chain.

\section{A. Varying Backoff Exponents}

In the proposed A-CSMA/CA scheme in Section III, $C W(i, s)$ is defined as a general function of $i$ and $s$. Here, we aims to explore the effects of different possible backoff schemes with respect to $s$ on the achieved throughput. Figure 4 show the saturation throughput when exponential backoff is adopted. More specifically, $C W(i, s)=C W_{\min } 2^{i} \beta^{s}$ where $\beta$ is called the channel backoff factor. Figure 4 demonstrate the throughput for $\beta=2,3$ and 4 , in comparison with the basic CSMA/CA (i.e., $\beta=1$ ). As can be observed, the larger $\beta$, the higher achieved throughput. This is simple to explain since the larger $\beta$ implies stronger preference towards the STAs with good channel states. Consequently, this prioritizing can improve the overall throughput of the network.

\section{B. Varying $C W_{\min }$}

The minimum contention window $C W_{\min }$ is a key parameter in the A-CSMA/CA protocol that significantly affects the achievable throughput. This is mainly because a larger $C W_{\min }$ can reduce the collision probability, while a smaller $C W_{\min }$ can reduce the collision avoidance overhead. To investigate the effects of $C W_{\min }$, Figures 5 illustrates the saturation throughput of the A-CSMA/CA scheme with $\beta=4$.

As shown in Figure 5, when the number of STAs in the WLAN is 1 , the achievable throughput decreases as $C W_{\text {min }}$ increases. This is due to the fact that the larger $C W_{\min }$ means a longer expected backoff time before each transmission, and thus, the lower achievable throughput. However, for $N=5$ and $N=40$, the throughput is first increasing and eventually falls down after a certain point. This happens because a larger contention window can also provide a better collision 


\begin{tabular}{|c|c|c|c|c|c|c|c|c|}
\hline SNR Range & {$[6,9)$} & {$[9,11)$} & {$[11,14)$} & {$[14,17)$} & {$[17,20)$} & {$[20,23)$} & {$[23,26)$} & {$[26, \infty)$} \\
\hline Data Rate (Mbps) & 6 & 9 & 12 & 18 & 24 & 36 & 48 & 54 \\
\hline
\end{tabular}

TABLE I: SNR ranges and corresponding data rates used in the illustrative results

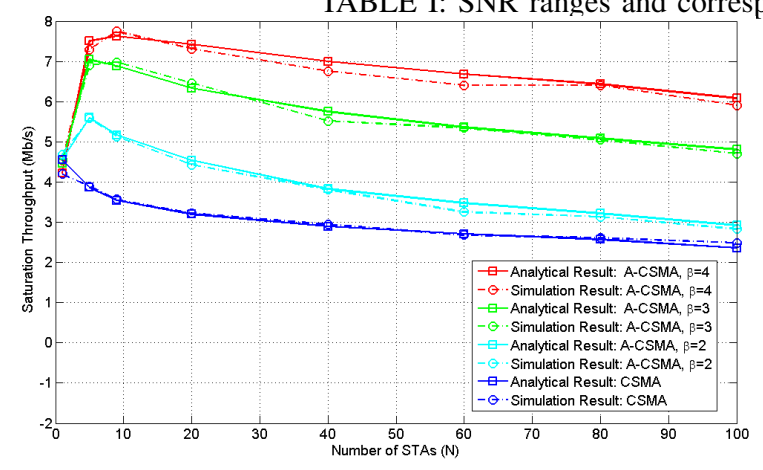

Fig. 4: Throughput vs. number of STAs for different $\beta$

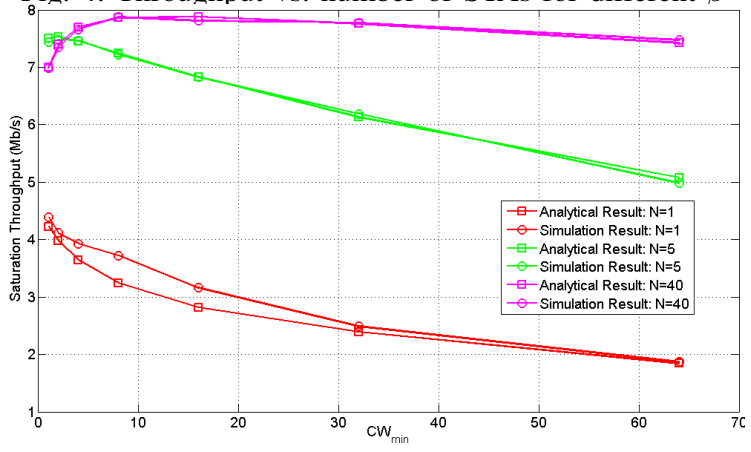

Fig. 5: Throughput vs. $C W_{\min }$ for different number of STAs

resolution among the STAs. Thus, to achieve the maximum throughput, it is clear that $C W_{\min }$ needs to be designed as a function of the number of STAs in the network. For instance, the optimal value of $C W_{\min }$ is around 4 for a network with 5 STAs, while $C W_{\min }=8$ gives a better throughput performance when $N=40$.

\section{Varying Transmission Duration $T$}

Figure 6 shows the system saturation throughput versus $T$ for CSMA and A-CSMA. For A-CSMA/CA, we set $\beta=4$ and $C W_{\min }=1$. In addition, the coherence time $L_{b}$ is set to $200 \mathrm{~ms}$ and $T$ is varied from $1 \mathrm{~ms}$ to $200 \mathrm{~ms}$. It is evident that A-CSMA/CA achieves higher throughput than CSMA/CA. As $T$ increases, the throughput of CSMA/CA first increases fast and then slows down, which is not exactly the case for A-CSMA/CA. The throughput of A-CSMA/CA first increases in $T$ (from $0 \mathrm{~ms}$ to $60 \mathrm{~ms}$ ). But, further increase in $T$ will decrease the achievable throughput. This is because the proposed A-CSMA/CA protocol prioritizes users based on observation of the current channel state. But, if $T$ is large relative to the value of coherence time, when the STA gets an opportunity for transmission, its current channel condition might already be different from the channel condition when the priority decision was made. That is why the throughput of ACSMA/CA decreases as $T$ increases after a certain threshold. This result suggests that the value of $T$ should optimally be selected according to the channel coherence time.

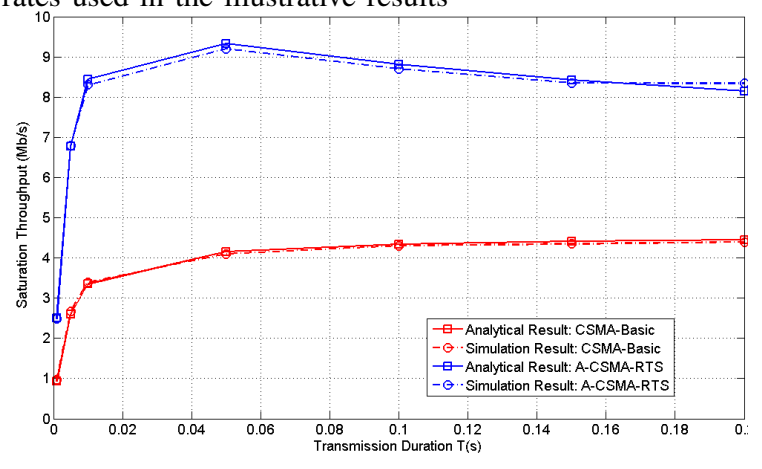

Fig. 6: Throughput vs. T

VI. CONCLUSION

This paper presents an adaptive CSMA/CA scheme to improve throughput by slightly modifying the random backoff mechanism of IEEE 802.11 DCF. As a result, throughput is improved since STAs with better channel qualities are given higher priority to transmit. To evaluate the performance of the proposed A-CSMA/CA protocol, a three-dimensional Markov chain is established to model the state transition of an STA. Illustrative simulation and analytical results confirm the precision of the proposed Markov chain model and also performance gain, i.e., high throughput, offered by the proposed A-CSMA/CA-based access strategy in comparison to conventional CSMA/CA.

\section{REFERENCES}

[1] L. Kleinrock and F. Tobagi, "Packet switching in radio channels: Part I-carrier sense multiple-access modes and their throughput-delay characteristics," IEEE Trans. Commun., vol. 23, no. 12, pp. 1400-1416, Dec. 1975.

[2] J. Zhang, Y. Chen, and I. Marsic, "MAC scheduling using channel state diversity for high-throughput IEEE 802.11 mesh networks," IEEE Commun. Mag., vol. 45, no. 11, pp. 94-99, Nov. 2007.

[3] T. Mishima, S. Miyamoto, S. Sampei, and W. Jiang, "Novel DCF-based multi-user MAC protocol and dynamic resource allocation for OFDMA WLAN systems," in Proc. IEEE Int. Conf. on Computing, Networking and Communications (ICNC), Jan. 2013, pp. 616-620.

[4] C.-S. Hwang, K. Seong, and J. Cioffi, "Opportunistic p-persistent CSMA in wireless networks," in Proc. IEEE Intl. Conf. Commun. (ICC), vol. 1, Jun. 2006, pp. 183-188.

[5] Y. Zhang, X. Zhang, and D. Yang, "Performance analysis of channelaware multichannel CSMA in wireless networks," in Proc. IEEE Veh. Tech. Conf. (VTC), May 2008, pp. 1657-1660.

[6] D.-Y. Yang, T.-J. Lee, K. Jang, J.-B. Chang, and S. Choi, "Performance enhancement of multirate IEEE 802.11 WLANs with geographically scattered stations," IEEE Trans. Mobile Comput., vol. 5, no. 7, pp. 906919, Jul. 2006.

[7] D. R. Chen and Y. Zhang, "Distributed MAC strategy for exploiting multi-user diversity in multi-rate IEEE 802.11 wireless LANs," in Proc. IEEE Int. Conf. on Mobile Adhoc and Sensor Systems (MCSS), Oct. 2007, pp. 1-11.

[8] M. Derakhshani and T. Le-Ngoc, "Adaptive access control of CSMA/CA in wireless LANs for throughput improvement," in Proc. IEEE Global Commun. Conf. (GLOBECOM), Dec. 2013, pp. 2951-2955.

[9] —-, "Cognitive MAC designs: Background," in Cognitive MAC Designs for OSA Networks. Springer International Publishing, 2014, pp. $15-31$.

[10] G. Bianchi, "Performance analysis of the IEEE 802.11 distributed coordination function," IEEE J. Sel. Areas Commun., vol. 18, no. 3, pp. 535-547, Mar. 2000. 